\title{
A pesquisa sobre envelhecimento humano: grupos de pesquisa no estado de São Paulo
}

\author{
Augusto Aldori Pedroso
}

\section{Resumo}

A produção de conhecimento sobre o envelhecimento humano estabelece um processo influenciado recentemente pela população acadêmica. Dessa forma, os grupos de pesquisa interessados nessa temática buscam perspectivas futuras para os idosos. Dependendo dessas perspectivas, as suas ações poderão ter diferentes influências para a população. Certamente, o mapeamento dos grupos de pesquisa contribuirá para o entendimento deste estudo. Assim, este artigo objetiva realizar uma cartografia do estudo sobre o envelhecimento humano no estado de São Paulo, a partir de fontes que relacionam pesquisadores do Diretório dos Grupos de Pesquisa no Brasil (2012) do Conselho Nacional de Desenvolvimento Científico e Tecnológico (CNPq). O caminho escolhido para a identificação foi a análise dos grupos que estudam o processo do envelhecimento humano no estado de São Paulo. A procura na base de dados deu-se por palavras-chave correlacionadas com esse processo. Assim, identificamos 48 grupos, 109 linhas de pesquisa e 212 pesquisadores, além das grandes áreas e áreas do conhecimento nas quais esses grupos estão inseridos, bem como o período de sua criação e sua dis- tribuição institucional. Os resultados apontam a natureza recente desse estudo com início de sua formação na década de 1980, sendo localizada, a maior parte dos grupos de pesquisa, basicamente, nas cidades de São Paulo e Campinas. Essa ocorrência pode ter relação com os programas de pós-graduação que essas duas cidades alocam e, também, pela titulação de seus líderes.

Palavras-chave: Cartografia. Envelhecimento humano. Grupos de pesquisa. Linhas de pesquisa.

\section{Introdução}

Neste último século, o aumento nas frequências absolutas e relativas de idosos relacionados aos grupos etários mais jovens levou a sociedade a passar por drásticas transformações. O elemento causador não é somente a queda das taxas de fecundidade e mortalidade, mas também é formado pelos avanços na produção de conhecimento e na aplicação de tecnologias visando à melhoria

* Graduado em Educação Física pela Faculdade Metodista de Santa Maria (FAMES) e Mestre em Envelhecimento Humano pela Universidade de Passo Fundo (UPF) - augustoaap@hotmail.com;

$\rightarrow$ http://dx.doi.org/10.5335/rbceh.2013.2806 
das condições de vida. Assim, mudanças no perfil demográfico populacional são impulsionadas para um novo contexto (CRUZ; SCHWANKE, 2001).

Diante desse novo panorama, surge a necessidade de uma dedicação maior dos pesquisadores na produção de conhecimentos relacionados ao estudo do envelhecimento humano. Esse anseio tem provocado questionamentos, expresso pelas seguintes perguntas: o que se estuda e se produz no interior dos grupos de pesquisa referente ao tema no estado de São Paulo? Quais são os estabelecimentos de ensino que abrigam esses grupos? Quantos são os grupos de pesquisas que tratam desse tema? Onde estão localizados esses grupos? Quais as áreas do conhecimento que estão pesquisando sobre envelhecimento humano? Quais as grandes áreas do conhecimento em que estão inseridos esses grupos?

Dessa forma, na perspectiva de atender a esses questionamentos, este trabalho propõe-se a realizar uma cartografia do estudo do envelhecimento humano no estado de São Paulo. Para isso, realizamos um esboço com a intenção de conhecer melhor o universo da pesquisa com base em fontes que relacionam pesquisadores que compõem o Diretório dos Grupos de Pesquisa no Brasil (2012) do Conselho Nacional de Desenvolvimento Científico e Tecnológico (CNPq).

Com a intenção de melhor compreender a proposta desse artigo, na introdução, contextualizamos o tema e o propósito do trabalho; seguimos com a metodologia empregada no estudo; nos resultados e discussões, concentramo-nos no período de criação dos grupos de pesquisa, nas grandes áreas e nas áreas do conhecimento predominantes, na distribuição dos grupos de pesquisa e nas instituições de ensino no estado, Nas considerações finais, retomamos os resultados mais relevantes e encerramos com as referências bibliográficas empregadas na proposta.

\section{Metodologia}

Na busca de subsídios que pudessem dar suporte metodológico para o presente estudo, adotamos como elemento norteador o trabalho de Pedroso e Diehl(2012), que publicaram o artigo "Cartografia do estudo sobre o envelhecimento humano no estado do Rio Grande do Sul". Os autores analisaram os grupos e linhas de pesquisa que estudam a temática no estado do Rio Grande do Sul, o período de criação, a distribuição geográfica, institucional, as grandes áreas do conhecimento e a área predominante.

As informações utilizadas na pesquisa dos autores foram reunidas a partir do Diretório dos Grupos de Pesquisa no Brasil, na versão 5.0, desenvolvida pelo Conselho Nacional de Desenvolvimento Científico e Tecnológico (CNPq), em 2012. O critério que os autores usaram para o reconhecimento dos grupos que compõem a massa crítica foi de pelo menos uma linha de pesquisa, conforme definido pelo pesquisador líder de cada grupo.

Em nossa pesquisa, adotamos também a versão 5.0 do Diretório dos Grupos de Pesquisa do CNPq. A primeira tentativa foi identificar os grupos de pesquisa do estado de São Paulo que mantêm alguma linha de pesquisa relati- 
va ao estudo do envelhecimento humano, por meio do campo atribuído à área do conhecimento registrada pelo líder de cada grupo. No entanto, o escopo não foi alcançado, pois a árvore de informações apresentada pelo Diretório não possibilita nenhuma associação a qualquer área relacionada ao envelhecimento humano, gerontologia ou geriatria.

É oportuno ressaltar que a Coordenação de Aperfeiçoamento de Pessoal de Nível Superior (Capes, 2012) inclui na grande área de conhecimento um item denominado de "multidisciplinar", o qual, certamente, tem a intenção de alocar os programas de pós-graduação que não se encaixam em nenhuma das áreas estabelecidas. Dessa forma, os cursos em nível de mestrado e doutorado em envelhecimento humano, gerontologia e geriatria, recomendados e reconhecidos, e em atividade no país, situam-se em uma subdivisão denominada de "área interdisciplinar".

O exposto acima não é exclusividade dos cursos que tratam da temática do envelhecimento humano, mas, também, é de outros programas que se enquadram de maneira semelhante, citando como exemplo a biotecnologia e o ensino e materiais.

Dessa maneira, rejeitada a via metodológica de investigar a partir das grandes áreas e seus setores, buscamos uma nova alternativa. No campo de busca de dados do Diretório, é possível realizar a pesquisa por palavras-chave, escolhendo também a unidade da federação.

Assim, passamos a empregar as expressões mais usuais na literatura científica em associação com a temática do envelhecimento humano e realizamos a pesquisa nos seguintes campos do Diretório: unidade da federação do grupo e consulta por palavras-chave no campo dos grupos.

Nessa perspectiva, foram utilizadas na pesquisa, as seguintes palavras-chave: envelhecimento humano, gerontologia, geriatria, velho(a)(s), idoso(a)(s), velhice, terceira idade, gerontogeriatria e, por fim, aposentadoria.

O início desse procedimento para a coleta dos dados ocorreu no mês de outubro de 2012, possibilitando a identificação de 56 grupos de pesquisa no estado de São Paulo. A leitura e a avaliação desses resultados (estando inclusos os campos: nome do grupo, palavras-chave e unidade da federação do grupo) permitiram sua análise de forma quali e quantitativa (BERKEY, 1996).

Sendo assim, identificou-se alguns grupos que não fazem parte do interesse desse estudo, devido aos "falsos positivos". Destinamos essa expressão, pois, na busca por palavras-chave, surgiram assuntos que tiveram resultado positivo, mas após uma análise, constatamos que tratava-se de outro assunto não ligado ao tema, caracterizando-se como falso, a exemplo da temática o envelhecimento de vinho" ou outras similares. Quando presentes nos campos utilizados na busca, foram eliminados da pesquisa. Desse modo, foram mantidos os grupos que, com segurança, fazem parte da temática investigada.

Os procedimentos de busca que utilizamos poderão ter deixado de fora do nosso estudo alguns grupos de pesquisa que trabalham efetivamente a temática abordada. Conjecturamos, por razões 
como o uso das palavras-chave utilizadas pelos líderes no formulário eletrônico do $\mathrm{CNPq}$, as quais podem ter sido mais específicas e diferentes daquelas que utilizamos em nosso estudo.

Finalizamos, assim, a primeira etapa com uma base de dados de 48 grupos de pesquisa no estado de São Paulo, que mantêm no mínimo uma linha de pesquisa voltada ao estudo do envelhecimento humano. É importante ressaltar, existem grupos com dedicação exclusiva ao tema, assim como existem grupos que estão com seu foco voltado para outras áreas, mas desenvolvem estudos relativos ao processo do envelhecimento humano.

A segunda etapa do método usado para o levantamento das informações sobre os grupos de pesquisa incidiu da diferenciação de dois subconjuntos. Primeiramente, os denominamos de "específicos", os quais têm o estudo do envelhecimento humano como tema central, ou seja, todas as suas linhas de pesquisa são referentes ao estudo dos idosos. Esses grupos perfazem um total de 23 no estado de São Paulo.

O segundo subconjunto é aquele que nomeamos de "não específicos", cujo elemento central de seus estudos não é o estudo do envelhecimento humano, mas contêm pelo menos uma linha de pesquisa com referência a esse estudo. Esses foram encontrados em um total de 25 no estado.

Nos 48 grupos encontrados, entre os subconjuntos específicos e não específicos, estão contidas 109 linhas de pesquisa referentes ao estudo do envelhecimento humano. Assim, para os grupos denominados específicos, foram encontradas 45 linhas de pesquisa, ficando as 64 linhas de pesquisa restantes para os não específicos. Todos os grupos registraram, por meio de seus líderes, palavras-chave que utilizamos na procura.

\section{Resultados e discussões}

Para subsidiar a prática da pesquisa sobre o envelhecimento humano, muitos grupos de pesquisa têm surgido no estado de São Paulo. Na Tabela 1, é apresentado o levantamento sobre o ano de criação dos grupos de pesquisa que estudam a temática ao longo das últimas décadas.

Tabela 1. Distribuição dos grupos de pesquisa conforme o período de criação, São Paulo, 2012

\begin{tabular}{lrrrrrrr}
\hline \multicolumn{8}{c}{$\begin{array}{c}\text { Grupos com linha de pesquisa referente ao } \\
\text { envelhecimento humano }\end{array}$} \\
\hline \multicolumn{1}{c}{ Específicos } & Não específicos & \multicolumn{2}{c}{ Total } \\
\hline $1985 / 1989$ & 2 & 8,7 & - & - & 2 & 4,2 \\
$1990 / 1994$ & 2 & 8,7 & 2 & 8,0 & 4 & 8,3 \\
$1995 / 1999$ & 3 & 13,0 & 7 & 28,0 & 10 & 20,8 \\
$2000 / 2004$ & 7 & 30,4 & 5 & 20,0 & 12 & 25,0 \\
$2005 / 2012$ & 9 & 39,1 & 11 & 44,0 & 20 & 41,7 \\
Total & 23 & 100,0 & 25 & 100,0 & 48 & 100,0 \\
\hline
\end{tabular}

Fonte: CNPq - Diretório dos Grupos de Pesquisa, Brasil, versão 5.0, 2012.

Analisando a Tabela 1, percebe-se que o marco inicial no estado de São Paulo pode ser considerado o ano de 1985 , coincidindo com os dados encontrados no estado do Rio Grande do Sul. Entretanto, é necessária precaução na análise dessas informações. Tendo em vista que o fato de o grupo ter se originado em um determinado ano não significa necessa- 
riamente que a produção científica tenha se iniciado nesse mesmo ano. Na busca realizada no Diretório, em São Paulo, não houve a recuperação de dados para os anos anteriores a 1985.

A criação desses grupos a partir do ano de 1985 combina com a contextualização histórica da década de 1980, quando a Capes (Comissão de Aperfeiçoamento de Pessoal de Nível Superior) determinava a vinculação dos grupos de pesquisas aos programas de pós-graduação das instituições de ensino superior, possibilitando, assim, o ingresso dos estudantes orientados naquele programa (Capes, 2012).

Foi na década de 1980, ainda, que ocorreram mudanças significativas na gerontologia brasileira. Essa ocorrência foi consequência do Plano Internacional de Ação para o Envelhecimento datado de 1982, conscientizando os países a colocar nas suas diretrizes, propostas que pudessem, de certa forma, garantir um envelhecimento com qualidade de vida. (KLETEMBERG et al., 2010).

A partir de Assembleia ocorrida em Viena, os países em desenvolvimento, como é o caso do Brasil, foram alertados a refletir sobre o impacto do envelhecimento populacional em suas sociedades (KLETEMBERG et al., 2010). Esse tema acendeu uma preocupação em diversos segmentos profissionais nas últimas décadas, levando o Brasil e, consequentemente, os estados brasileiros, como São Paulo, a aumentarem os programas destinados a essa população (KLETEMBERG et al., 2010).

Em consequência dessa atenção voltada aos idosos, concomitantemente com os esforços dos profissionais que voltaram os seus interesses para essa área, percebeu-se o aumento da formação de grupos de estudos e pesquisa. Assim, o tema envelhecimento humano começou a gerar conhecimento (GOLDSTEIN, 1999).

Cronologicamente, na década de 1990, acontece no estado de São Paulo a formação de mais 14 grupos com linhas de pesquisa referentes a esse processo. Nesse período, ocorre a mudança da percepção dos pesquisadores sobre esse segmento populacional. A visão de outrora, que associava o envelhecimento com os problemas sociais e dependência, passa a reconhecer essa população, conferindo-lhe o direito ao bem-estar e à autonomia (KLETEMBERG et al., 2010).

O esboço acima tem coerência com a perspectiva apontada por Cachioni (1999, p. 161) ao estudar a formação das universidades da terceira idade no Brasil. O autor relata que, "a partir dos anos de 1990, as universidades começam a abrir as suas portas tanto para o contingente idoso, quanto para os profissionais interessados no estudo desse processo". Com isso, começam a ser ofertados os programas de ensino, saúde e lazer, com o intuito de combinar sociabilidade e educação permanente.

Conforme a Tabela 1, ao analisar-se os grupos específicos, observa-se que cinco deles foram formados na década de 1990, ocorrendo uma expansão em números a partir de 2000. Considerando esses dados, fica visível e confirmado que a produção de conhecimento sobre o envelhecimento humano é muito recente $\mathrm{e}$ intensa, tanto no país, quanto no estado de São Paulo. 
Observa-se, também, que a partir do ano de 2000 até o ano de 2011, foram criados 32 grupos de pesquisa no estado. Esses números correspondem a $66,6 \%$ do total dos grupos encontrados que fazem parte do presente estudo, entre os quais 16 são específicos e 16 não específicos.

Outro fator relevante foi a criação de cursos de pós-graduação que tratam do tema. A Pontifícia Universidade Católica de São Paulo (PUC/SP) foi a pioneira no estado de São Paulo, com a concepção do Programa de Mestrado em Gerontologia sendo um curso multidisciplinar, criado em 1997 (CNPq, 2012). Outra universidade em destaque é a Universidade Estadual de Campinas (Unicamp), que instituiu seus cursos de Mestrado em 2008 e Doutorado em 2010, também em Gerontologia (CNPq, 2012).

A partir da criação desses cursos de pós-graduação, consolidaram-se, cada vez mais, as agregações de profissionais que trabalham e estudam esse processo intrincado do envelhecer, repercutindo, dessa maneira, um avanço gradativo na área da pesquisa e na criação de grupos de pesquisa (PRADO; SAYD, 2004).

Grandes áreas do conhecimento predominantes

As grandes áreas podem ser entendidas como a "aglomeração de diversas áreas do conhecimento em virtude da afinidade de seus objetos, métodos cognitivos e recursos instrumentais refletindo contextos sociopolíticos específicos” (CAPES, 2012, p. 1). Essas áreas proporcionam aos órgãos de ciência e tecnologia uma maneira rápida e funcional de acrescentar informações com respeito ao desenvolvimento científico e tecnológico, principalmente aqueles referentes a projetos de pesquisa e recursos humanos. A classificação das áreas do conhecimento foi realizada de forma hierárquica, abrangendo todos os níveis, dos gerais aos específicos, começando com 8 grandes áreas do conhecimento, 76 áreas e 340 subáreas (CAPES, 2012).

No preenchimento do formulário eletrônico do CNPq, entre os vários campos existentes, o líder de cada grupo informa a grande área predominante, juntamente com a área de atuação a qual o grupo encontra-se vinculado. Define-se, dessa maneira, o lugar do grupo nos diversos campos do conhecimento.

Essa investigação comprovou que os grupos de pesquisa que estudam o envelhecimento humano no estado de São Paulo estão presentes em quatro das grandes áreas do conhecimento, pois nas outras grandes áreas não houve recuperação de grupos com interesse nessa temática. Cabe salientar que no estado do Rio Grande do Sul repetem-se as mesmas grandes áreas, com exceção das Ciências Exatas (PEDROSO; DIEHL, 2012).

A Tabela 2 mostra a distribuição dos grupos de pesquisa nas grandes áreas do conhecimento, segundo os critérios que escolhemos: grupos específicos e não específicos. 
Tabela 2. Distribuição dos grupos de pesquisa nas grandes áreas do conhecimento, São Paulo, 2012

\begin{tabular}{|c|c|c|c|c|c|c|}
\hline \multirow{3}{*}{ Grandes áreas } & \multicolumn{6}{|c|}{ Grupos com linha de pesquisa referente ao envelhecimento humano } \\
\hline & \multicolumn{2}{|c|}{ Grupos específicos } & \multicolumn{2}{|c|}{ Grupos não específicos } & \multicolumn{2}{|c|}{ Total } \\
\hline & $\mathrm{n}$ & $\%$ & $\mathrm{n}$ & $\%$ & $\mathrm{n}$ & $\%$ \\
\hline Ciências da Saúde & 14 & 60,9 & 16 & 64,0 & 30 & 62,5 \\
\hline Ciências Sociais & 6 & 26,1 & 2 & 8,0 & 8 & 16,7 \\
\hline Ciências Humanas & 2 & 8,7 & 7 & 28,0 & 9 & 18,8 \\
\hline Ciências Exatas & 1 & 4,3 & - & - & 1 & 2,1 \\
\hline Total & 23 & 100,0 & 25 & 100,0 & 48 & 100,0 \\
\hline
\end{tabular}

Fonte: CNPq- Diretório dos Grupos de Pesquisa, Brasil, versão 5.0, 2012.

Analisando a Tabela 2, fica evidente a predominância das Ciências da Saúde entre as grandes áreas dos grupos que estudam o envelhecimento humano no estado de São Paulo. Do total dos grupos que pertencem às Ciências da Saúde, os denominados específicos apresentam um percentual de $60,8 \%$. Já os não específicos são responsáveis por $64 \%$ das pesquisas. Nota-se, também, um contingente importante, porém, em menor número de grupos pertencentes às Ciências Sociais, perfazendo um total de $26 \%$ dos específicos e $8 \%$ dos não específicos.

No estudo realizado por Pedroso e Diehl (2012), também ficou clara a liderança das Ciências da Saúde. Segundo os autores, a predominância dessa ciência está em parte relacionada com algum grau de interesse de cunho nacional, como políticas, intervenções e comunicação, entre outros.

No estado de São Paulo, como no estudo realizado no Rio Grande do Sul, o elemento explicativo para esse episódio, certamente, é a repercussão dos programas de pós-graduação que estudam o envelhecimento humano, em nível de mestrado e doutorado. Por serem multidisciplinares e pela diversidade de áreas, grande parte dos seus pesquisadores pertence à área da saúde.

Nota-se, também, que em São Paulo as Ciências Humanas aparecem em segundo lugar, tanto nos grupos específicos quanto nos não específicos. O estudo de Pedroso e Diehl (2012) revela que o estado do Rio Grande do Sul mantém esse mesmo perfil.

Área do conhecimento predominante

Entende-se como área do conhecimento "o conjunto de informações inter-relacionadas, coletivamente, construídas, reunidas segundo a natureza do objeto de investigação com finalidades de ensino, pesquisa e aplicações práticas" (CAPES, 2012, p. 1).

No estado de São Paulo, a pesquisa identificou a formação de grupos de pesquisa interessados no envelhecimento humano em 15 áreas predominantes, conforme nos indica a Tabela 3. 
Tabela 3. Distribuição dos grupos de pesquisa segundo áreas predominantes, São Paulo, 2012

\begin{tabular}{|c|c|c|c|c|c|c|}
\hline \multirow{3}{*}{ Área predominante } & \multicolumn{6}{|c|}{$\begin{array}{c}\text { Grupos com linha de pesquisa referente ao } \\
\text { envelhecimento humano }\end{array}$} \\
\hline & \multicolumn{2}{|c|}{ Específicos } & \multicolumn{2}{|c|}{ Não específicos } & \multicolumn{2}{|c|}{ Total } \\
\hline & $\mathrm{n}$ & $\%$ & $\mathrm{n}$ & $\%$ & $\mathrm{n}$ & $\%$ \\
\hline Antropologia & - & - & 1 & 3,8 & 1 & 2,2 \\
\hline Arquitetura e Urbanismo & - & - & 1 & 3,8 & 1 & 2,2 \\
\hline Ciências da Computação & 1 & 4,8 & - & - & 1 & 2,2 \\
\hline Comunicação & 1 & 4,8 & - & - & 1 & 2,2 \\
\hline Educação & - & - & 4 & 15,4 & 4 & 8,7 \\
\hline Educação Física & - & - & 2 & 7,7 & 2 & 4,3 \\
\hline Enfermagem & 1 & 4,8 & 3 & 11,5 & 4 & 8,7 \\
\hline Fisioterapia e Terapia Ocupacional & 2 & 9,5 & 2 & 7,7 & 4 & 8,7 \\
\hline Fonoaudiologia & 1 & 4,8 & 1 & 3,8 & 2 & 4,3 \\
\hline Medicina & 4 & 19,0 & 3 & 11,5 & 7 & 15,2 \\
\hline Nutrição & - & - & 1 & 3,8 & 1 & 2,2 \\
\hline Odontologia & 1 & 4,8 & 2 & 7,7 & 3 & 6,5 \\
\hline Psicologia & 2 & 9,5 & 2 & 7,7 & 4 & 8,7 \\
\hline Saúde Coletiva & 3 & 14,3 & 4 & 15,4 & 7 & 15,2 \\
\hline Serviço Social & 5 & 23,8 & 1 & 3,8 & 6 & 13,0 \\
\hline Total & 21 & 100,0 & 26 & 100,0 & 46 & 100,0 \\
\hline
\end{tabular}

Fonte: CNPq - Diretório dos Grupos de Pesquisa, Brasil, versão 5.0, 2012.

A Tabela 3 evidencia que, entre as áreas predominantes, a Medicina, juntamente com a Saúde Coletiva, destaca-se tanto entre os grupos denominados específicos quanto entre os não específicos. Embora em dimensões próprias, o estudo referente aos grupos de pesquisa no estado do Rio Grande do Sul apresenta perfil semelhante, porém com liderança somente da Medicina (PEDROSO; DIEHL, 2012).
Distribuição dos grupos de pesquisa no estado de São Paulo

Os dados contidos na Tabela 4 mostram que $35,4 \%$ dos grupos de pesquisa do estado de São Paulo estão concentrados na capital. Essa ocorrência é confirmada no perfil geral da base de dados do CNPq, incluindo todos os grupos existentes no estado, sejam eles com relação ao envelhecimento humano ou não. 
Tabela 4. Distribuição dos grupos de pesquisa segundo as cidades. São Paulo 2012

\begin{tabular}{|c|c|c|c|c|c|c|}
\hline \multirow{3}{*}{ Municípios } & \multicolumn{6}{|c|}{ Grupos com linha de pesquisa referente ao envelhecimento humano } \\
\hline & \multicolumn{2}{|c|}{ Específicos } & \multicolumn{2}{|c|}{ Não específico } & \multicolumn{2}{|c|}{ Total } \\
\hline & $\mathrm{n}$ & $\%$ & $\mathrm{~N}$ & $\%$ & $\mathrm{n}$ & $\%$ \\
\hline Araçatuba & - & - & 1 & 4,0 & 1 & 2,1 \\
\hline Araraquara & 1 & 4,3 & - & - & 1 & 2,1 \\
\hline Araras & - & - & 1 & 4,0 & 1 & 2,1 \\
\hline Barretos & - & - & 1 & 4,0 & 1 & 2,1 \\
\hline Bauru & 2 & 8,7 & - & - & 2 & 4,2 \\
\hline Botucatu & 1 & 4,3 & 1 & 4,0 & 2 & 4,2 \\
\hline Campinas & 1 & 4,3 & 5 & 20,0 & 6 & 12,5 \\
\hline Franca & 1 & 4,3 & - & - & 1 & 2,2 \\
\hline Marília & - & - & 3 & 12,0 & 3 & 6,3 \\
\hline Piracicaba & - & - & 1 & 4,0 & 1 & 2,1 \\
\hline Ribeirão Preto & 1 & 4,3 & 3 & 12,0 & 4 & 8,3 \\
\hline Rio Claro & - & - & 1 & 4,0 & 1 & 2,1 \\
\hline Santo André & - & - & 1 & 4,0 & 1 & 2,1 \\
\hline Santos & - & - & 1 & 4,0 & 1 & 2,1 \\
\hline São Carlos & 3 & 13,0 & - & - & 3 & 6,2 \\
\hline São Paulo & 13 & 56,5 & 4 & 16,0 & 17 & 35,4 \\
\hline Taubaté & - & - & 2 & 8,0 & 2 & 4,2 \\
\hline Total & 23 & 100,0 & 25 & 100,0 & 48 & 100,0 \\
\hline
\end{tabular}

Fonte CNPq - Diretório dos Grupos de Pesquisa, Brasil, versão 5.0, 2012.

Esse perfil também guarda similaridade com os estudos de Pedroso e Diehl (2012), que apontam que os grupos de pesquisa que focam o tema residem basicamente na capital.

Um aspecto muito importante a ser considerado no estado de São Paulo éque em segundo lugar destaca-se a cidade de Campinas, com o percentual de $12,5 \%$. O motivo das duas cidades, São Paulo e Campinas, estarem em evidência pode estar associado à existência das Instituições de Ensino Superior (IES) com os cursos de pós-graduação em Envelhecimento Humano/Gerontologia.
Distribuição dos grupos de pesquisa nas Instituições de Ensino Superior do estado de São Paulo

Os dados da Tabela 5 evidenciam que 171 das instituições de ensino superior paulistas trazem no seu interior grupos de pesquisa que desenvolvem pelo menos uma linha de pesquisa relacionada à temática do envelhecimento humano. Destaca-se, entre elas, a Universidade Estadual Paulista (UNESP), liderando com um percentual de $20,8 \%$ dos grupos. 
Tabela 5. Distribuição dos grupos de pesquisa segundo as Instituições de Ensino Superior, São Paulo, 2012

\begin{tabular}{|c|c|c|c|c|c|c|}
\hline \multirow{3}{*}{ Instituições } & \multicolumn{6}{|c|}{ Grupos com linha de pesquisa referente ao envelhecimento humano } \\
\hline & \multicolumn{2}{|c|}{ Específicos } & \multicolumn{2}{|c|}{ Não específico } & \multicolumn{2}{|c|}{ Total } \\
\hline & $\mathrm{n}$ & $\%$ & $\mathrm{n}$ & $\%$ & $\mathrm{n}$ & $\%$ \\
\hline UNICAMP & 1 & 4,3 & 5 & 20,0 & 6 & 12,5 \\
\hline UNIABC & - & - & 1 & 4,0 & 1 & 2,1 \\
\hline USC & 1 & 4,3 & - & - & 1 & 2,1 \\
\hline UNESP & 4 & 17,4 & 6 & 24,0 & 10 & 20,8 \\
\hline FEB & - & - & 1 & 4,0 & 1 & 2,1 \\
\hline UNIMES & - & - & 1 & 4,0 & 1 & 2,1 \\
\hline UFSCAR & 3 & 13,0 & - & - & 3 & 6,3 \\
\hline UNIARARAS & - & - & 1 & 4,0 & 1 & 2,1 \\
\hline USP & 6 & 26,1 & 4 & 16,0 & 10 & 20,8 \\
\hline UNINOVE & 1 & 4,3 & - & - & 1 & 2,1 \\
\hline PUC-SP & 5 & 21,7 & 1 & 4,0 & 6 & 12,5 \\
\hline FCMSCSP & 1 & 4,3 & - & - & 1 & 2,1 \\
\hline FUABC & - & - & 1 & 4,0 & 1 & 2,1 \\
\hline UNITAU & - & - & 2 & 8,0 & 2 & 4,2 \\
\hline USJT & 1 & 4,3 & - & - & 1 & 2,1 \\
\hline UNIMEP & - & - & 1 & 4,0 & 1 & 2,1 \\
\hline IAL & - & - & 1 & 4,0 & 1 & 2,1 \\
\hline Total & 23 & 100,0 & 25 & 100,0 & 48 & 100,0 \\
\hline
\end{tabular}

Fonte CNPq- Diretório dos Grupos de Pesquisa, Brasil, versão 5.0, 2012.

Cabe salientar que existem vários campi dessa instituição em diferentes cidades no estado. Já a Pontifícia Universidade Católica de São Paulo (PUC-SP) aparece como vice-líder, juntamente com a Universidade Estadual de Campinas (Unicamp), com um percentual de 12,5\% dos grupos. Contudo, com peso relativamente importante, surge a Universidade Federal de São Carlos (UFSCar) com $6,2 \%$ do total investigado.
Considerações finais

O propósito deste trabalho foi realizar um mapeamento do estudo do envelhecimento humano no estado de São Paulo. Para isso, foi investigado o tema procurando revelar as áreas e as grandes áreas do conhecimento predominantes, a distribuição dos grupos de pesquisa e instituições de ensino que realizam esse estudo, assim como o período de criação desses grupos. 
A pesquisa sobre essa população acontece, principalmente, na área das ciências da saúde, com importante participação das ciências humanas. As ciências sociais e exatas também desempenham papel importante nas pesquisas. Certamente, o tema interessa a pesquisadores de muitos campos da produção de conhecimento.

Os grupos de pesquisa operantes no estado de São Paulo aglomeram-se, basicamente, na capital e na cidade de Campinas, onde desenvolvem as suas pesquisas. Com isso, envolvem, principalmente, a Pontifícia Universidade Católica de São Paulo (PUC-SP) e a Universidade Estadual de Campinas (Unicamp). Em algum nível, essa característica está agregada aos programas de pós-graduação abrigados nessas instituições. Concomitantemente à concentração existente, a pesquisa sobre o tema está distribuída em várias IES. Não se trata, porém, de um conjunto uniforme. Existem grupos que voltam seus estudos especificamente para o envelhecimento humano, os quais perfazem um percentual de 47,9\% do total da investigação. Os $52 \%$ restantes ficam com os grupos denominados não específicos. A visão das ciências da saúde e das ciências humanas é aqui muito mais marcante, materializada, principalmente, por meio da medicina, saúde coletiva, serviço social, odontologia e terapia ocupacional.

Dessa maneira, podemos concluir que o estudo do envelhecimento humano no estado de São Paulo é de natureza recente. No entanto, ocorreu uma grande expansão na pesquisa sobre os idosos e o processo do envelhecimento humano no estado, principalmente a partir do ano 2000. Tais acréscimos, julgamos, estão ligados à expansão da Universidade Aberta da Terceira Idade (UnATI) e à constituição de novos grupos de pesquisa.

Esta proposta de investigação não engloba toda a temática do estudo do envelhecimento humano no estado, dessa forma, esse assunto será retomado em outra oportunidade, procurando apresentar uma nova abordagem do tema. Assim, acreditamos que a relevância deste trabalho consiste em fornecer, para a comunidade acadêmica em geral e interessados, uma visão detalhada do estudo sobre o envelhecimento humano no estado de São Paulo.

\section{The research on human aging: research} groups in the state of São Paulo

\section{Abstract}

The production of knowledge about the human aging process establishes a recently influenced by the academic population. Thus, the research groups interested in this issue seek future prospects for the elderly. Depending on these perspectives, their actions may have different influences for this population. Certainly, the mapping of the research groups will contribute to the understanding of this study. Thus, this paper aims to conduct a study on the mapping of the Human Aging in the state of São Paulo, from sources that relate researchers Directory of Research Groups in Brazil (2012) National Council for Scientific and Technological Development (CNPq). The path chosen for the analysis was the identification of groups who study the process of Human Aging in the state of São Paulo. 
The search in the database was given by keywords correlated with this process. Thus, we identified 48 groups, 109 lines of research and 212 researchers, in addition to large areas and areas of knowledge that these groups are inserted, and the period of its creation and its institutional distribution. The results indicate the nature of this study with recent onset of its formation in the years 80 . Most research groups are located primarily in the cities of São Paulo and Campinas. This occurrence may be consistent with programs graduate in these two cities allocate and also by titration of their leaders.

Keywords: Mapping. Human aging. Research Groups. Research Lines.

\section{Referências}

CACHIONI, M. Universidades da terceira idade: das origens à experiência brasileira. In: NERI, A. L.; DEBERT, G. G. (Org.). Velhice e sociedade. Campinas: Papirus, 1999. p. 141-178.

CAPES. Coordenação de Aperfeiçoamento de Pessoal de Nível Superior. 2012. Disponível em: <http://www.capes.gov.br/>. Acesso em: 15 set. 2012.

CRUZ I. B. M, SCHWANKE C. H. A. Reflexões sobre biogerontologia como uma ciência generalista, integrativa e interativa. Rev. de Estudos Interdisciplinares sobre Envelhecimento 2001, n. 3, p. 7-36.

CNPq. Conselho Nacional de Desenvolvimento Científico e Tecnológico. Diretório dos Grupos de Pesquisa do Brasil [portal na Internet] 2012. Disponível em: <http://www. cnpq.br/>. Acesso em: 11 set. 2012.

BERKEY D. B. Meaning and value of oral health for older person: research findings and clinical care implications. A reactor's notes. Getodontology, 13(2): 90-93, Dec. 1996.
GOLDSTEIN, L. L. A Produção Científica Brasileira na Área da Gerontologia: (19751999). Revista on-line Biblioteca Prof. Joel Martins, Curitiba, v. 1, n. 1, out. 1999.

KLETEMBERG, D. F.; et al. A construção histórica do conhecimento da enfermagem gerontológica no Brasil. Revista de Enfermagem, Rio de Janeiro, v. 14, n. 4, p. 787-796, out./dez. 2010.

PEDROSO, A. A.; DIEHL. A. A. Cartografia do estudo sobre o envelhecimento humano no estado do Rio Grande do Sul. 2012. Revista de Divulgação. Disponível em: <http:// portaldoenvelhecimento.org.br/revista/index. php/revistaportal/article/viewFile/238/260>. Acesso em: 20 maio 2012.

PRADO, S. D.; SAYD, J. D. A pesquisa sobre envelhecimento humano no Brasil: grupos e linhas de pesquisa. Ciência \& Saúde Coleti$v a$, Rio de Janeiro, v. 9, n. 1, p. 57-68, 2004. 